Document downloaded from:

http://hdl.handle.net/10251/153040

This paper must be cited as:

Revert, A.; Calm, R.; Vehí, J.; Bondía Company, J. (2011). Calculation of the Best BasalBolus Combination for Postprandial Glucose Control in Insulin Pump Therapy. IEEE Transactions on Biomedical Engineering. 58(2):274-281. https://doi.org/10.1109/TBME.2010.2058805

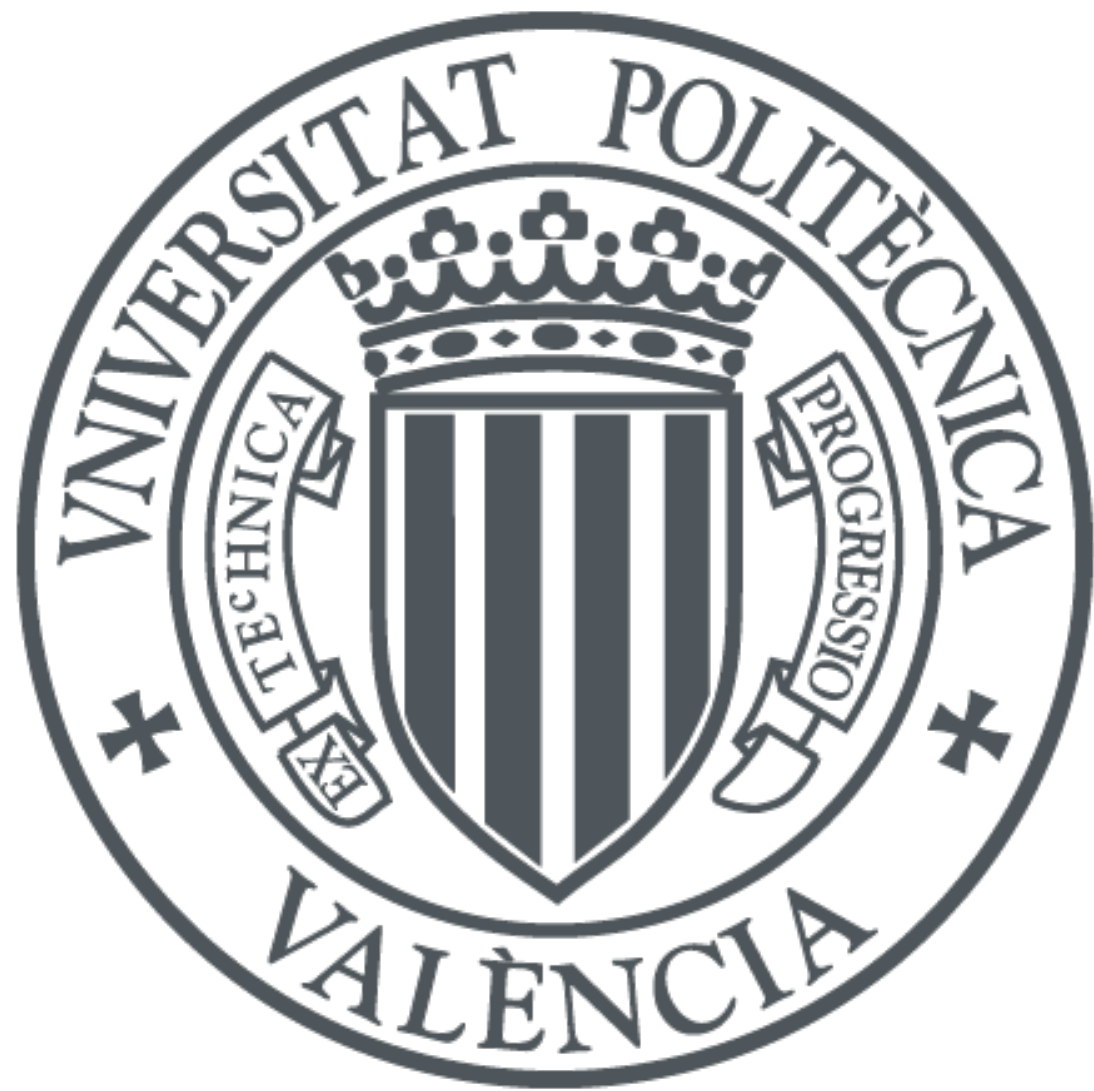

The final publication is available at

https://doi.org/10.1109/TBME.2010.2058805

Copyright Institute of Electrical and Electronics Engineers

Additional Information

"(C) 2011 IEEE. Personal use of this material is permitted. Permissíon from IEEE must be obtained for all other uses, in any current or future media, including reprinting/republishing this material for advertisíng or promotional purposes, creating new collective works, for resale or redistribution to servers or lists, or reuse of any copyrighted component of this work in other works." 


\title{
Calculation of the best basal-bolus combination for postprandial glucose control in insulin pump therapy
}

\author{
A. Revert, R. Calm, J. Vehí, J. Bondia*
}

\begin{abstract}
Intensive insulin therapy in type 1 diabetes is based on the well-established practice of adjusting basal and bolus insulin independently. Basal insulin delivery is designed to optimize glucose concentrations between meals and overnight, while bolus insulin delivery is designed to optimize postprandial glucose concentrations. However, this strategy shows some limitations in the postprandial glucose control, especially for meals with high carbohydrate content. Strategies based on coordinating basal and bolus insulin in the postprandial period help in overcoming these limitations. An algorithm, based on mathematically guaranteed techniques (interval analysis), is presented in this work. It determines, given the current glycemic state of the patient and the meal to be ingested, a basalbolus combination that will yield a tight postprandial glycemic control according to the International Diabetes Federation guidelines. For a given meal, the algorithm reveals which bolus administration mode will enable a good postprandial performance: standard, square-wave, dual-wave or temporal basal decrement. The algorithm is validated through an in silico study using the 30 subjects in the educational version of the FDA-accepted UVa simulator.
\end{abstract}

Index Terms - type 1 diabetes, insulin pump therapy, postprandial glucose control, interval analysis, set inversion, optimal experiment design.

\section{INTRODUCTION}

I $\mathrm{N}$ the 1990s, the Diabetes Control and Complications Trial [1] showed that any improvement in the glucose control as measured by the level of $\mathrm{HbA} 1 \mathrm{c}$, leads to a reduction of the risk to suffer chronic complications associated with diabetes. For this reason, euglycemia has been established as the control objective for patients with type 1 diabetes mellitus, except if some contraindication exists. This is accomplished through the use of exogenous insulin, promoting the transport and storage of blood glucose into the cell and thus decreasing blood glucose concentration.

Intensive insulin therapy is based on the administration of basal and bolus insulin. Basal insulin delivery is designed to optimize glucose concentrations between meals and overnight, while bolus insulin delivery is designed to counteract the hyperglycemia occurring after meals.

J. Bondia and A. Revert are with the Instituto Universitario de Automática e Informática Industrial, Universidad Politécnica de Valencia, C/ Camino de Vera, s/n, 46022 Valencia, Spain

R. Calm and J.Vehí are with the Institut d'Informàtica i Aplicacions, Universitat de Girona, Campus de Montilivi, Edifici P-IV, Girona, Spain

*corresponding author, e-mail: jbondia@isa.upv.es
In the case of multiple daily injections, this is implemented with the use of long-acting insulin analogues for basal insulin, expected to produce a flat profile of plasma insulin concentration along the day, and rapid-acting insulin formulations for bolus insulin, expected to have a fast onset of action to overcome postprandial hyperglycemia. In the case of continuous subcutaneous insulin infusion, the same rapid-acting insulin is used for both basal and bolus. In this case, insulin pumps allow for the application of smart insulin infusion profiles. Currently, basal infusion rates are programmed with time-varying profiles over a 24-hour period, in order to account for circadian variations of patient's insulin sensitivity and activity. Bolus insulin infusion can be delivered via different profiles as well (standard, extended/square wave, combination/dual wave) to deal with different meal compositions or other circumstances such as stress and/or exercise [2].

In [3] it was shown that standard therapies that consider only bolus insulin to control postprandial glucose can not be enough to fulfill the International Diabetes Federation (IDF) guidelines (no hypoglycemia and two-hour postprandial glucose below $140 \mathrm{mg} / \mathrm{dL}$ ) [4]. Taking advantage of the possibilities that insulin pumps provide, the smart prandial insulin profiles can be utilized to overcome the limitations of the standard therapies, as demonstrated in [3].

In this work, an algorithm based on mathematically guaranteed techniques (interval analysis) to calculate the best prandial basal-bolus combination leading to a tight postprandial glucose control is presented. In particular, a set inversion problem is posed to determine, for a given meal, which prandial insulin administration mode and dose will yield a good postprandial performance, according to the patient's model. Section II reviews the main theoretical concepts related to interval analysis and set inversion, presenting the Set Inversion Via Interval Analysis (SIVIA) algorithm and the definitions needed to understand it. The new methodology for prandial insulin determination is introduced in Section III. Section IV presents an optimal experiment design procedure for patient's model identification. Finally, Section V presents an in silico validation using the FDA-accepted UVa simulator [5].

\section{INTERVAL ANALYSIS AND SET INVERSION}

Interval analysis arose in the context of numerical analysis and the study of propagation of computational errors in finite number systems [6], [7]: if real numbers are substituted by compact subsets of the digital scale (intervals) 
which contain it, and real operators by interval operators, computations will lead to intervals that contain the actual solution, whose width is a measure of the approximation error. It is precisely this property of inclusion of the actual solution that makes interval analysis and methods derived very interesting when a mathematical guarantee is desired.

Inclusion functions are thus one of the fundamental tools in interval analysis.

In the following, $[x]$ will denote a real interval, and $\underline{x}, \bar{x}$ are its left and right endpoints. Interval vectors, or boxes, will be denoted in boldface, $[\mathbf{x}]$. The set of all real intervals will be denoted by $\mathbb{I}$ and the set of $n$-dimensional boxes as $\mathbb{\mathbb { R } ^ { n }}$.

A formal definition follows.

Definition II.1 Given a function $\mathbf{f}: \mathbb{R}^{n} \rightarrow \mathbb{R}^{m}$, the interval function $[\mathbf{f}]: \mathbb{R}^{n} \rightarrow \mathbb{R}^{m}$ is an inclusion function for $\mathbf{f}$ if for any box $[\mathbf{x}]=[\underline{\mathbf{x}}, \overline{\mathbf{x}}] \in \mathbb{I}^{n}$

$$
[\mathbf{f}]([\mathbf{x}]) \supseteq\left[\min _{\mathbf{x} \in[\mathbf{x}]} \mathbf{f}(\mathbf{x}), \max _{\mathbf{x} \in[\mathbf{x}]} \mathbf{f}(\mathbf{x})\right] .
$$

The simplest way to get an inclusion function for $\mathbf{f}$ is replacing the real variable $x$ with an interval variable $[x]$ and the real arithmetic operations with corresponding interval operations. The result $[\mathbf{f}]$ is called a natural inclusion function of $\mathbf{f}[6]$. However, this may yield significant overestimation when multiple instances of a variable appear in the expression to evaluate (multiincidences problem). Other inclusion functions have been studied to reduce this problem like centered forms or Taylor expansion forms. See for instance [6]-[9] for more details on this topic.

Currently, interval analysis is a mature technology that has been successfully applied in fields aside numerical analysis such as robotics, control, computer graphics, economy, global optimization, and fault detection, among others [9].

An important application of interval analysis is the solution of set inversion problems. Let $\mathbb{X} \subseteq \mathbb{R}^{n}$ and $\mathbb{Y} \subseteq \mathbb{R}^{m}$ be an input and output space, respectively. Given a set $\mathcal{Y} \subseteq \mathbb{Y}$ and a map $\mathbf{f}: \mathbb{X} \rightarrow \mathbb{Y}$, the set $\mathcal{X}:=\{\mathbf{x} \in \mathbb{X} \mid \mathbf{f}(\mathbf{x}) \in \mathcal{Y}\}$ is sought. The set $\mathcal{Y}$ is usually defined through constraints on the output space. The SIVIA algorithm [9] makes use of a branch-andbound technique together with interval analysis to get an approximation of the solution set $\mathcal{X}$. This approximation is done in terms of subpavings (collection of boxes of the appropriate dimension with non-overlapping interiors). An inner and outer subpaving, which will be denoted as $[\mathcal{X}]_{\mathrm{i}}$ and $[\mathcal{X}]_{\mathrm{o}}$ respectively, are built so that $[\mathcal{X}]_{\mathrm{i}} \subseteq \mathcal{X} \subseteq[\mathcal{X}]_{\mathrm{o}}$. Hence, it is guaranteed that $[\mathcal{X}]_{\mathrm{i}}$ will contain only solutions while the complementary set of $[\mathcal{X}]_{\mathrm{o}}$, denoted as $\overline{[\mathcal{X}]_{\mathrm{o}}}$, will contain only non-solutions (see Fig. 1).

Some previous definitions follow before presenting the SIVIA algorithm.

Definition II.2 The width of a box $[\mathbf{x}]=[\underline{\mathbf{x}}, \overline{\mathbf{x}}] \in \mathbb{R}^{n}$ is $w([\mathbf{x}]):=\max _{i \in\{1, \ldots, n\}}\left(\overline{x_{i}}-\underline{x_{i}}\right)$.

Definition II.3 The midpoint of a box $[\mathbf{x}]=[\underline{\mathbf{x}}, \overline{\mathbf{x}}] \in \mathbb{R}^{n}$ is $m([\mathbf{x}]):=(\underline{\mathbf{x}}+\overline{\mathbf{x}}) / 2$.

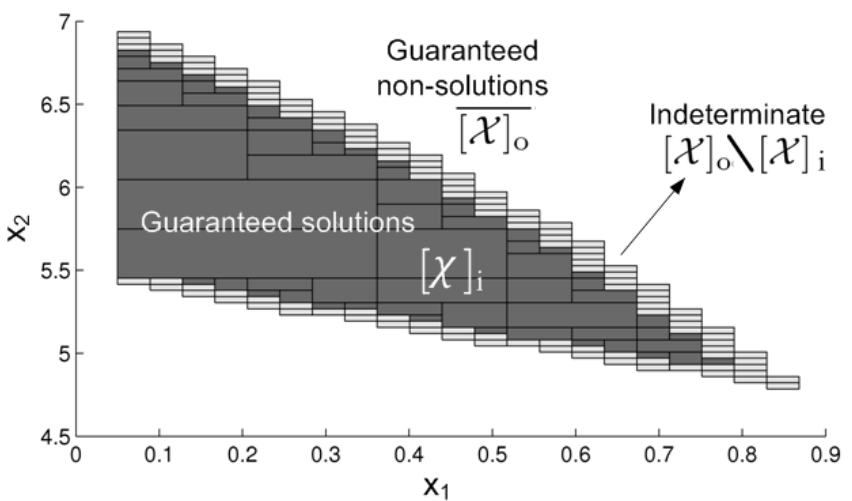

Fig. 1. Plot that illustrates the concept of inner and outer subpaving. The dark rectangles represent the inner subpaving and guarantee the fulfillment of the constraints. The outer subpaving is made up of both the dark and the light rectangles. Its complementary set (in white) is guaranteed to contain only non-solutions that violate some of the constraints. Results in the boundary (light rectangles) are unknown a priori.

Definition II.4 The left and right children of a box $[\mathbf{x}]=$ $[\underline{\mathbf{x}}, \overline{\mathbf{x}}] \in \mathbb{R}^{n}$ are

$L([\mathbf{x}]):=\left[\underline{x_{1}}, \overline{x_{1}}\right] \times \cdots \times\left[x_{j}, m\left(\left[x_{j}\right]\right)\right] \times \cdots \times\left[\underline{x_{n}}, \overline{x_{n}}\right]$

$R([\mathbf{x}]):=\left[x_{1}, \overline{x_{1}}\right] \times \cdots \times\left[\bar{m}\left(\left[x_{j}\right]\right), \overline{x_{j}}\right] \times \cdots \times\left[x_{n}, \overline{x_{n}}\right]$

where $j$ is the first component of $[\mathbf{x}]$ with maximum width, that is, $j=\min \left\{i \mid w\left(\left[x_{i}\right]\right)=w([\mathbf{x}])\right\}$.

Algorithm II.1 (SIVIA, [9]) Let $\mathcal{X}$ be the solution set sought and $[\mathcal{X}]_{\mathrm{i}}$ and $[\mathcal{X}]_{\mathrm{o}}$ be two subpavings corresponding to inner and outer approximations of $\mathcal{X}$ as defined above. Let $[t]: \mathbb{R}^{n} \rightarrow \mathbb{I B}$ be a test interval function from the set of $n$-dimensional interval vectors (box in the input space) to the set of interval booleans, $\mathbb{I B}=\{0,1,[0,1]\}$ (where 0 stands for false, 1 for true and $[0,1]$ for indeterminate). Finally, let $[\mathbf{x}] \in \mathbb{I}^{n}$ be an initial box in the input space and $\epsilon$ be a positive precision factor that can be chosen arbitrarily low. The SIVIA algorithm is as follows:

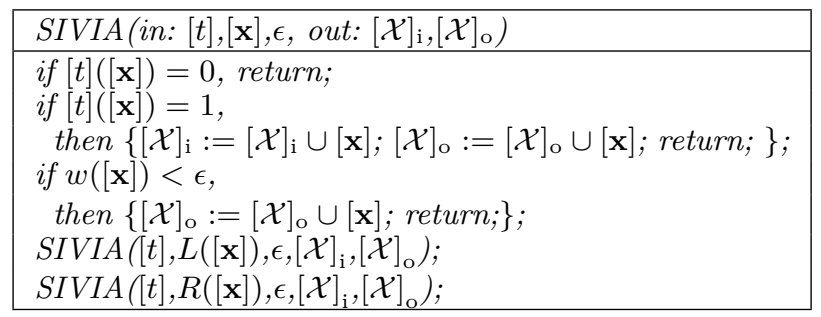

The inner subpaving will thus consist of the boxes classified as true, while the outer subpaving contains the true and indeterminate boxes (of width smaller than the tolerance defined). Not small enough indeterminate boxes will be splitted in two subboxes by the midpoint of its largest dimension and the procedure repeated.

\section{Set-InVERsion-BASEd PRANDial Insulin DELIVERY}

With a proper instantiation of the input and output spaces, $\mathbb{X}$ and $\mathbb{Y}$, and the test interval function $[t]$, SIVIA 


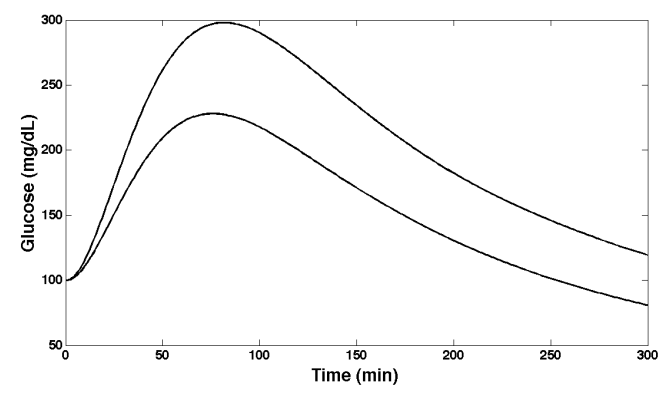

Fig. 2. Output of an interval simulation. Upper and lower envelopes include all possible glucose responses for the input box.

can be used to gain insight on the different dosage strategies that can be applied depending on the patient and the nature of the meal and to select the best basal-bolus combination that will yield a good postprandial control.

For this purpose, the following set inversion problem is posed:

The input space $\mathbb{X}$ corresponds to the bolus insulin dose, the modified basal insulin infusion at meal time and the time of restoration of basal to its baseline value.

For a given box in the input space, $[\mathbf{x}]$, and a set of constraints $\mathcal{C}$ on postprandial glycemia, the test function $[t]([\mathbf{x}])$ will determine whether: (1) all the insulin therapies contained in $[\mathbf{x}]$ fulfill the constraints $\mathcal{C}$ (True case); (2) none of the insulin therapies contained in $[\mathbf{x}]$ fulfill the constraints $\mathcal{C}$ (False case); (3) some of the therapies in $[\mathbf{x}]$ fulfill the constraints, while others do not (Indeterminate case).

The constraints $\mathcal{C}$ are defined here following the IDF guidelines for postmeal control [4]: non-hypoglycemia and two-hour postprandial glucose value below $140 \mathrm{mg} / \mathrm{dL}$, in a five-hour time horizon. The hypoglycemic threshold is not explicitly defined in the guidelines. A value of $70 \mathrm{mg} / \mathrm{dL}$ is adopted here. Additionally, two extra constraints are defined: five-hour postprandial glucose value above $90 \mathrm{mg} / \mathrm{dL}$ and a maximum glucose slope of $0.05 \mathrm{mg} / \mathrm{dL} / \mathrm{min}$ starting four hours after the meal. These additional constraints are included to minimize both the risk of hypoglycemia after the first five hours and late undesirable glucose rebounds.

Finally, a patient's model is used to predict postprandial glycemia, $G$, that is compared against the above constraints. An interval simulation of the model is carried out using Modal Interval Analysis [10]. This allows to obtain tight bounds of the envelopes enclosing the collection of postprandial glucose profiles originated from the set of therapies in $[\mathbf{x}]$. For a given time $t,[G](t)$ will thus be an interval (see Fig. 2). A time step of 1 minute is used in the simulation.

Remark that the above additional constraints are imposed on the model predictions, which may be inaccurate after a few hours compared to the real patient behavior. Values were tuned accounting for this mismatch in the case of the model used here.

The test interval function, $[t]([\mathbf{x}])$ is thus defined as:

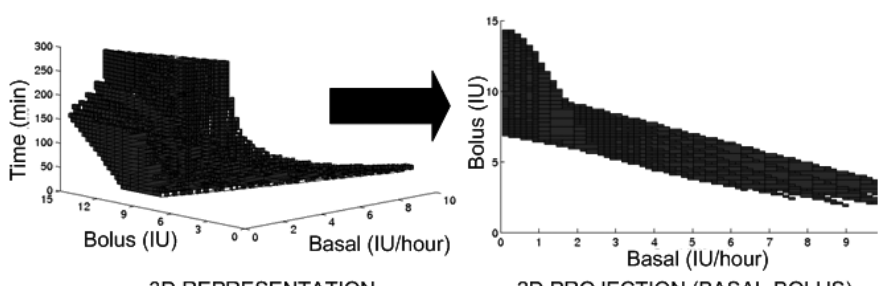

3D REPRESENTATION

Fig. 3. Plot that represents a 3D (basal, bolus and time) feasible set with its corresponding basal-bolus $2 \mathrm{D}$ projection.

True: $\quad\left(\forall t_{k} \in[0,300][G]\left(t_{k}\right) \geq 70\right) \wedge$

$\left(\forall t_{k} \in[120,300][G]\left(t_{k}\right) \leq 140\right) \wedge$

$[G](300) \geq 90) \wedge$

$\left(\forall t_{k} \in[240,300] \frac{[G]\left(t_{k+1}\right)-[G]\left(t_{k}\right)}{t_{k+1}-t_{k}} \leq 0.05\right)$

False: $\quad\left(\exists t_{k} \in[0,300][G]\left(t_{k}\right)<70\right) \vee$

$\left(\exists t_{k} \in[120,300][G]\left(t_{k}\right)>140\right) \vee$

$([G](300)<90) \vee$

$\left(\exists t_{k} \in[240,300] \frac{[G]\left(t_{k+1}\right)-[G]\left(t_{k}\right)}{t_{k+1}-t_{k}}>0.05\right)$

Indet.: otherwise

where $t_{k}$ is a discrete time instant. Remark that the above inequalities correspond to interval inequalities $([\underline{x}, \bar{x}] \leq \alpha \Leftrightarrow \bar{x} \leq \alpha,[\underline{x}, \bar{x}] \geq \alpha \Leftrightarrow \underline{x} \geq \alpha, \alpha \in \mathbb{R})$.

The kind of inner subpavings that are obtained after the application of the algorithm is shown in Fig. 3 (left). The subpaving consists of $3 \mathrm{D}$ feasible boxes, where these three dimensions correspond to the bolus dose, the postprandial basal dose and time of restoration of basal to baseline. The 2D basal-bolus projection (Fig. 3 right) contains information on the different basal-bolus combinations that will lead to a good performance for a particular patient and meal. In this way, combinations in the inner subpaving are guaranteed (with a proper selection of the time of restoration of basal to baseline) to yield a glucose profile that fulfills the defined constraints. On the contrary, combinations outside the outer subpaving will violate some constraint (see Fig. 4). If the outer subpaving is empty, there is no solution to the problem unless the constraints are relaxed.

The projected basal-bolus space can be divided into regions corresponding to different bolus administration modes present in current insulin pumps, plus a new one called here temporal basal decrement (see Fig. 5). This is especially important since it allows the automatic selection of the best administration mode. So far this is done based on the physician's heuristics. To ease interpretability, a normalization is done with respect to the patient's nominal basal and standard bolus from its insulin-to-carbohydrate ratio (I:C). The point $(1,1)$ corresponds thus to the standard therapy.

Fig. 5 shows a situation where all the different bolus administration modes (standard bolus, square bolus, dualwave bolus and temporal basal decrement) will result in 

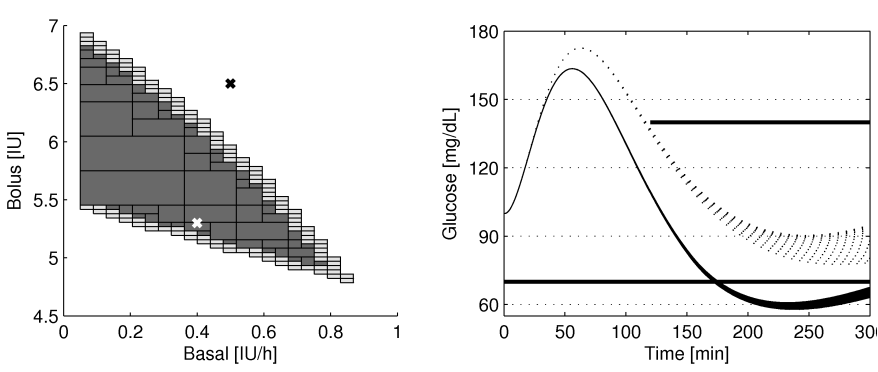

Fig. 4. Plot that shows the glucose response for a basal-bolus combination inside the inner subpaving (dotted line) and for a combination outside the outer subpaving (solid line). The glucose response has been represented for times of restoration from 30 to 300 min demonstrating that for combinations in the inner subpaving always exists at least one solution, selecting the appropriate time of restoration of basal from the feasible set, that fulfills the constraints whereas, for combinations outside the outer subpaving is impossible to obtain a proper glucose control.

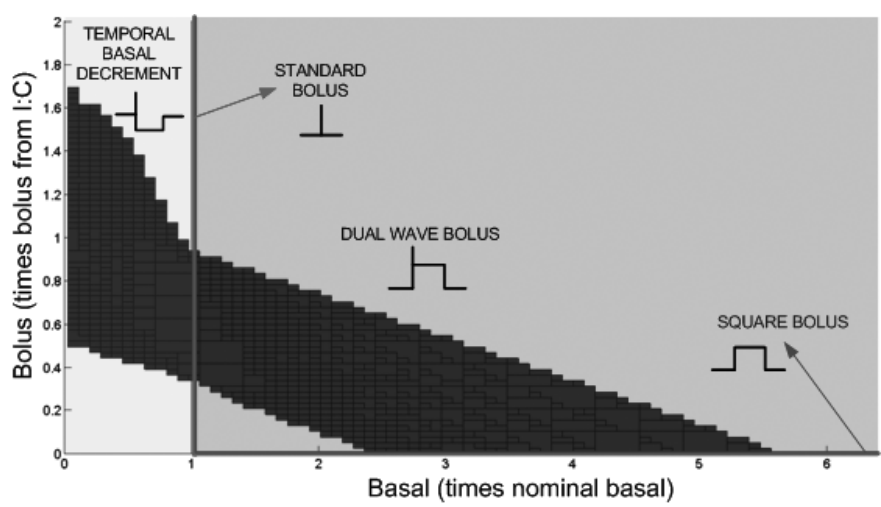

Fig. 5. Normalized feasible set that shows all the possible bolus administration modes. Therapies with nominal basal correspond to a standard strategy, therapies with an increment in basal postprandial dosage result in a dual-wave or square-wave strategy whereas therapies with less postprandial basal than baseline are called here as temporal basal decrement mode. The corresponding insulin infusion profiles are depicted for each region.

a good glucose response, fulfilling the IDF guidelines of postmeal control. Depending on the patient and on the grams of carbohydrates of the meal, the number of bolus modes fulfilling the constraints may be reduced.

The procedure used to select a specific point (basalbolus combination) from all the possible ones is done as follows. The 2D basal-bolus subpaving is divided into two smaller subpavings (when possible) corresponding to a positive and negative basal deviation from nominal. The first one will correspond to a bolus mode currently available in insulin pumps (standard/square/dual-wave). The second one will correspond to a new administration mode, found to be the only solution for big sized meals (see Section V). The optimal point of each of these new subpavings is selected to be the geometric centroid obtained by the expression:

$$
\hat{x}=\frac{\Sigma_{i} m\left(\left[\mathbf{x}_{i}\right]\right) A_{i}}{\Sigma_{i} A_{i}}
$$

where $m\left(\left[\mathbf{x}_{i}\right]\right)$ and $A_{i}$ are the centroids (midpoints) and areas, respectively, of the different subpaving boxes.
Once the 2D basal-bolus optimal point for each therapy has been selected, time of restoration of basal to baseline is chosen as the mean point of the interval of time that fulfills the constraints for this basal-bolus optimum (third dimension of the subpaving in Fig. 3).

\section{PATIENT'S MODEL IDENTIFICATION}

A critical issue in the above methodology is the identification of the patient's model. Due to the fact that clinical experiments are expensive and difficult to carry out, they have to be designed meticulously in order to obtain results that provide as much information as possible for the posterior parameter estimation process.

Optimal experiment design (OED) consists in identifying the proper experiment setup (selection of inputs to excite the process) that delivers experimental data allowing parameter estimation with minimal uncertainty. In this manner, OED helps in improving the a posteriori identification of the parameters, minimizing also problems of bad conditioning in their estimation [11], [12].

The first step in OED consists in selecting the parameters describing the experimental setup. The parameters considered here are:

- ingested carbohydrates,

- bolus insulin dosage, and

- time instant of bolus insulin infusion (before or after the meal).

The duration of each experiment (meal) is five hours and the number of measurements is fixed and determined by the frequency of the continuous glucose sensor (usually 5 minutes). Each experiment will be carried out on different days, approximately at the same time every day. In this case the experiments will take place at lunch time.

Bound constraints on the parameters are included to avoid impractical results. So, the grams of carbohydrates are limited to values between 50-100gr (corresponding to an acceptable range for the lunch) while the bolus insulin dosage must remain in the range $\pm 20 \%$ the patient's standard bolus for that meal. Infusion time for bolus insulin is limited from 30 minutes before the intake to 30 minutes after it. In addition, extra constraints are added to avoid hypoglycemia $(<70 \mathrm{mg} / \mathrm{dL})$ or extremely high hyperglycemia ( $>300 \mathrm{mg} / \mathrm{dL})$.

In order to reproduce a situation as real as possible during the in silico validation, a model structurally different from the one used by the UVa simulator is considered in this work, forcing model mismatch. The Hovorka model has been selected for this purpose [13]. A priori identifiable parameters reported in [13] will be considered for identification, with the exception of $F_{01}$ (non-insulindependent glucose flux) that is replaced with $t_{\max , G}$ (time to maximum carbohydrate absorption) so as to include at least one parameter related to the meal intake (see [13] for the model details). Moreover $Q_{2}(0)$ (initial mass of glucose in the non-accessible compartment) is also included as an additional parameter to be identified (see Table II). The values of the parameters used are mean parameters with 


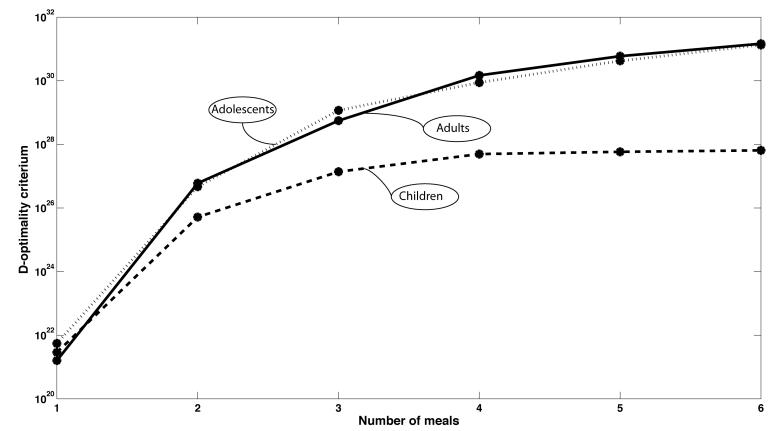

Fig. 6. Value of the D-optimality criterion against different number of meals for the three groups of patients.

the body weight set to the mean body weight of the adults, adolescents and children that will be used in the in silico validation. An insulin sensitivity proportionality factor is applied and fixed to $0.5,0.6$ and 0.7 respectively. Nominal basal is taken as the basal infusion normalizing glucose around $100 \mathrm{mg} / \mathrm{dL}$. I:C is estimated through simulations and fixed to 1:14, 1:19 and 1:25 for adults adolescents and children.

The optimality criterion used in this work is Doptimality, corresponding to the maximization of the determinant of the Fisher Information Matrix [11]. Fig. 6 shows the evolution of this criterion for the three groups of patients with respect to the number of days (number of lunches) used for identification. It can be observed that the improvement in the criterion resulting from adding a new day decreases progressively. So, a compromise solution between the time needed to identify and the a posteriori identifiability of the model has been selected. Therefore, data obtained from four different lunches will be used in the identification process.

Table I shows the OED results obtained for adults, adolescents and children. In general, it can be inferred from the OED that the best results in identification will be obtained by alternating the time of administration of the bolus insulin with respect to meal time, always distancing the administration as much as possible from the meal. Moreover, both carbohydrate grams and deviation from nominal I:C must be selected from the extreme values. That is, big or small meals will be better for identification than medium size meals. In the same way, the glucose response has to be excited as much as possible delivering bolus the further from nominal the better, but always within control limits to not decompensate the patient in excess. This fact can be clearly observed in the adults group whereas in adolescents and children, due to their higher insulin sensitivity, the possible violation of the bound constraints reduces the freedom in the selection of the experiment parameters.

A posteriori identifiability analysis of the model with the results obtained from Table I, proves that the seven to-be-identified parameters have a coefficient of variation below $10 \%$, fact that guarantees their identifiability.

The identification results obtained for one representative
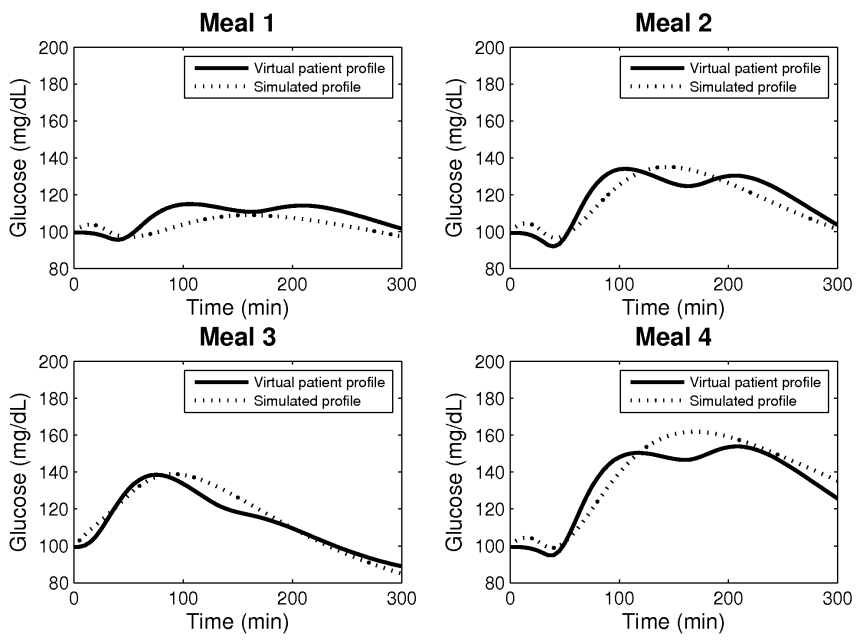

Fig. 7. Four-meal identification following the OED results. The solid line represents the glucose profile of the virtual patient whereas the dotted line represents the profile simulated by the identified model.

adult patient from the UVa simulator are shown in Fig. 7. The coefficient of determination, $R_{2}$, is $86 \%$ which assures a good identification quality.

\section{RESUlts AND VALIDATION}

The UVa simulator is used for an in silico validation of the methodology here presented. This simulator, widely described in [5], was accepted by the US Food and Drug Administration (FDA) as a substitute to animal trials in the preclinical testing of closed-loop control strategies in January, 2008.

The simulator provides a set of virtual subjects based on real individual data, a simulated sensor that replicates the typical errors of continuous glucose monitoring and a simulated insulin pump. In the educational version of the simulator 30 virtual subjects are available, including 10 children, 10 adolescents and 10 adults.

In this work, these 30 virtual patients are used. Patients' models are identified following the results obtained in Section IV. Basal baseline is taken as the basal infusion normalizing glucose around $100 \mathrm{mg} / \mathrm{dL}$. I:C ratio is obtained from the questionnaire available for each patient in the UVa simulator. Table II shows the average estimation of the parameters identified together with their coefficient of variation (inter-patient variability) for each of the groups of patients considered (adults, adolescents and children).

Once the model is obtained, the normalized 2D basalbolus projections are computed for meals in the range 40140 grams of carbohydrates and initial normoglycemia. Fig. 8 shows the results obtained for the same virtual patient as in Section IV.

The most remarkable issue extracted from Fig. 8 is that for low carbohydrate content meals $(<80 \mathrm{gr})$, different possible strategies lead to a good glucose control whereas as the carbohydrate content increases, the set of possible solutions is reduced, as expected. For big sized meals (> 100 gr), only a temporal basal decrement strategy with 
TABLE I

Proper FOUR MEALS EXPERIMENT SETUP ACCORDING TO THE OED

\begin{tabular}{|c|c|c|c|c|c|c|c|c|c|c|c|c|}
\hline & \multicolumn{4}{|c|}{ ADULTS } & \multicolumn{4}{|c|}{ ADOLESCENTS } & \multicolumn{4}{|c|}{ CHILDREN } \\
\hline & Meal 1 & Meal 2 & Meal 3 & Meal 4 & Meal 1 & Meal 2 & Meal 3 & Meal 4 & Meal 1 & Meal 2 & Meal 3 & Meal 4 \\
\hline cho(gr) & 50 & 100 & 50 & 100 & 100 & 50 & 90 & 100 & 100 & 70 & 50 & 100 \\
\hline$\triangle(I: C)$ & $20 \%$ & $20 \%$ & $20 \%$ & $-20 \%$ & $-20 \%$ & $20 \%$ & $0 \%$ & $20 \%$ & $20 \%$ & $17 \%$ & $20 \%$ & $-10 \%$ \\
\hline me $(\min )$ & -30 & -30 & 30 & -30 & -30 & -30 & 0 & -30 & -30 & 0 & -30 & -30 \\
\hline
\end{tabular}

TABLE II

MODEL PARAMETERS ESTIMATION

\begin{tabular}{|c|c|c|c|c|c|c|c|c|}
\multicolumn{1}{c}{} & \multicolumn{1}{c}{$t_{\max }, I$} & $t_{\max }, G$ & $S_{I T}^{f}$ & $S_{I D}^{f}$ & $S_{I E}^{f}$ & $E G P_{0}$ & $Q_{2}(0)$ \\
\hline \multirow{2}{*}{ ADULTS } & Mean & 60.85 & 61.17 & 0.0049 & 0.0034 & 0.0015 & 0.0137 & 47.55 \\
\cline { 2 - 9 } & CV & 0.27 & 0.23 & 0.98 & 1.13 & 2.20 & 0.35 & 0.37 \\
\hline \multirow{2}{*}{ ADOLESCENTS } & Mean & 53.39 & 59.45 & 0.0045 & 0.00873 & 0.0010 & 0,01590 & 20.84 \\
\cline { 2 - 9 } & CV & 0.47 & 0.29 & 1.43 & 1.25 & 2.26 & 0.30 & 0.27 \\
\hline \multirow{2}{*}{ CHILDREN } & Mean & 59.19 & 53.07 & 0.0203 & 0.0074 & 0.0160 & 0.0175 & 18.41 \\
\cline { 2 - 9 } & CV & 0.42 & 0.48 & 1.55 & 0.90 & 1.73 & 0.31 & 0.81 \\
\hline
\end{tabular}
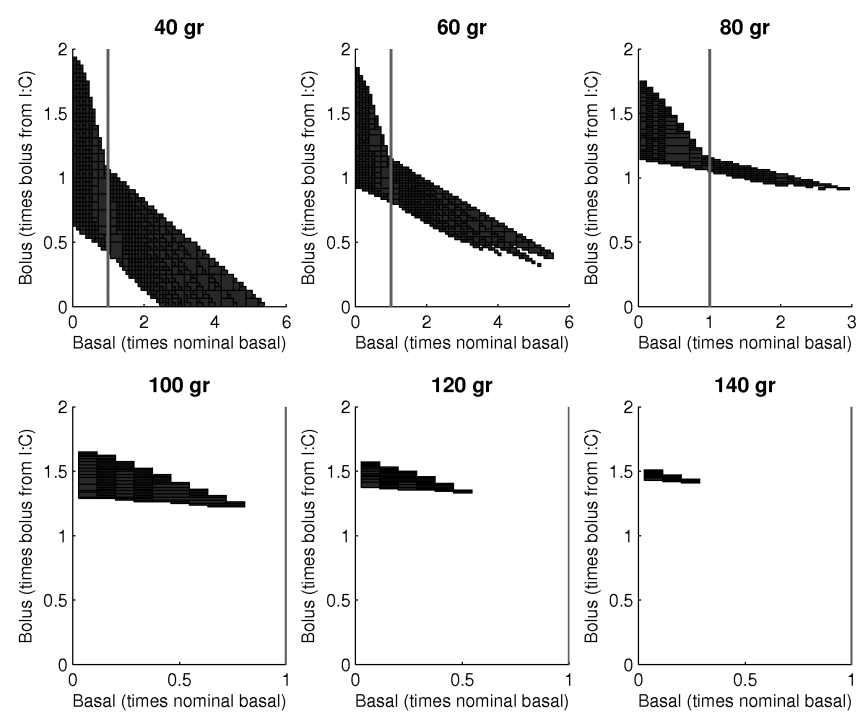

Fig. 8. 2D basal-bolus projection of normalized feasible sets for a meal of 40 to 140 grams of carbohydrates and initial normoglycemia. The vertical line stands for the standard strategy with basal equal to its baseline value.

an increment in the bolus dosage with respect to nominal ("a superbolus therapy" [14], [15]) can yield to a good postprandial control. Similar behavior can be observed in the rest of the patients being, the temporal basal decrement strategy the only strategy that provides good results for big sized meals. Occasionally, for a particular meal and patient, the feasible set could be empty, in which case the upper constraint will be relaxed in steps of 20 $\mathrm{mg} / \mathrm{dL}$ to a maximum of $300 \mathrm{mg} / \mathrm{dL}$.

In order to check the results obtained in Fig. 8, an intermediate sized meal (60 gr) and a big sized meal (120 gr) are selected to compare the glucose response applying the basal-bolus insulin combinations given by the algorithm for each possible bolus administration mode with the response using the standard therapy (see Fig. 9). For the 60 gr meal any of the three possible therapies (standard, dual-wave and temporal basal decrement) yields a good performance whereas for the $120 \mathrm{gr}$ meal, the basal-bolus combination given by the algorithm using a temporal basal decrement strategy improves significantly the performance of the standard therapy, incapable of fulfilling the IDF guideless for postprandial control. In this case, both strategies could produce a very mild hypoglycemia seven hours after the meal ${ }^{1}$. However, this is not at all critical.

Moreover, to compare the basal-bolus combination strategy presented here with the standard bolus strategy in the entire population, the control-variability grid analysis tool (CVGA) is used [16]. This tool provides a measure of the extreme glucose excursions observed using different therapies. Fig. 10 shows the shape of a CVGA plot, where the different zones correspond to different degrees of variability (see [16] for more details). The CVGA has been used to compare the glucose profile using the standard bolus strategy with the profile obtained with the temporal basal decrement bolus strategy in the three groups of virtual patients and both for small-intermediate sized meals (40, 60 and 80 gr) and big sized meals (100, 120 and $140 \mathrm{gr}$ ) in a seven-hour time horizon.

The dual-wave strategy results have been omitted because previous experiments showed that in many cases this strategy is not applicable (see Fig 8). For adolescents and children, the range of big sized meals has been reduced to 100-120 grams of carbohydrates, because, in general, the feasible set of prandial insulin infusions was empty for bigger sized meals. Additionally, the virtual child number 8 was eliminated from the study because again the feasible set was empty for meals higher than $60 \mathrm{~g}$ even relaxing constraints.

To improve the interpretability and due to the dispersion and quantity of the data, the results obtained by the CVGA are presented as histograms, the bars corresponding to the percentage of points falling in each of the

\footnotetext{
${ }^{1}$ There is no consensus about the hypoglycemic threshold. Some clinicians use a value of $60 \mathrm{mg} / \mathrm{dL}$. Here, a more restrictive value of $70 \mathrm{mg} / \mathrm{dL}$ was used.
} 

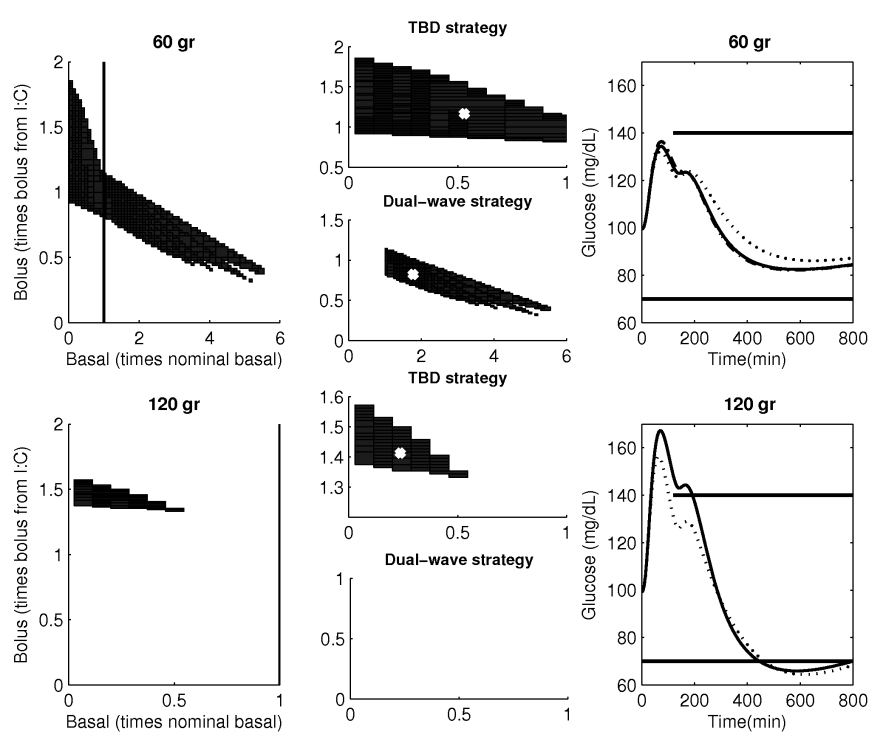

Fig. 9. Comparison among the postprandial glucose profiles applying different therapies for 60 and $120 \mathrm{gr}$ meals. The subpavings corresponding to each bolus administration mode are represented (when existing) separately. The specific basal-bolus combination selected for each strategy is showed in the subpavings as a thick dot. Finally, the glucose response using the basal-bolus combinations given by the algorithm and the standard therapy are plotted. The solid line corresponds to the standard therapy, computed from the I: $\mathrm{C}$ ratio given by the UVa simulator, whereas the dotted line and the dotteddashed line represent the temporal basal decrement (TBD) and the dual-wave therapy respectively. The horizontal lines represent the IDF constraints.

zones. Fig. 11 presents the results obtained by showing the percentage of points falling in $\mathrm{A}, \mathrm{B}, \mathrm{A}+\mathrm{B}, \mathrm{C}, \mathrm{D}$ and E zones.

It can be extracted from the figure that in every case the percentage of points falling in the $\mathrm{A}+\mathrm{B}$ zone using the temporal basal decrement strategy is higher than in the case of the standard bolus strategy increasing, in most cases, also the number of points falling in the A zone. In this way, for meals lower than 100 gr the percentage of points falling in the $\mathrm{A}+\mathrm{B}$ zone is higher than $70 \%$ for the three groups of patients, falling, in the case of the adults, $60 \%$ in the A zone, compared to $10 \%$ for the standard therapy. For big sized meals and specially for adolescents and children, this percentage decreases but, nevertheless, it doubles the percentage obtained with the standard therapy. Moreover, this strategy is also capable of decreasing the percentage of points falling in zone $\mathrm{E}$ (erroneous control).

It is worth pointing out that no information about the composition of the meals is available. So, in this in silico validation the composition of the meals has been taken into account implicitly during the model identification. In case of having information about the glucose response of the patients for different composition meals, an individual identification of the the gastrointestinal part of the model for each type of meal should be carried out. In this manner, depending on the composition of a given meal, one or another model should be used. The rest of the methodology would not experience any changes.

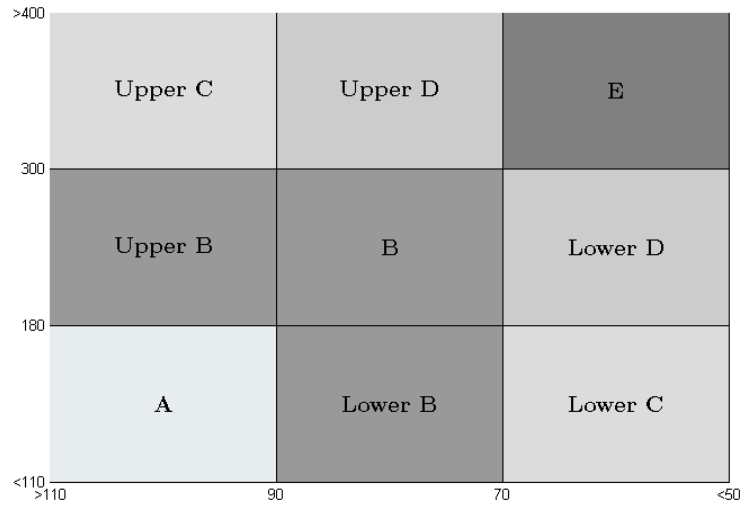

Fig. 10. Example of the CVGA grid appearance. Points falling in zone A represent an accurate control whereas zone $\mathrm{B}$ corresponds to benign control deviation. Zones $\mathrm{C}$ and $\mathrm{D}$ correspond to an overcorrection in the first case and a failure in the control in the second case, of hypo or hypoglycemia depending on whether the points fall in the lower or the upper zones respectively. Finally points falling in zone E represent an erroneous control.

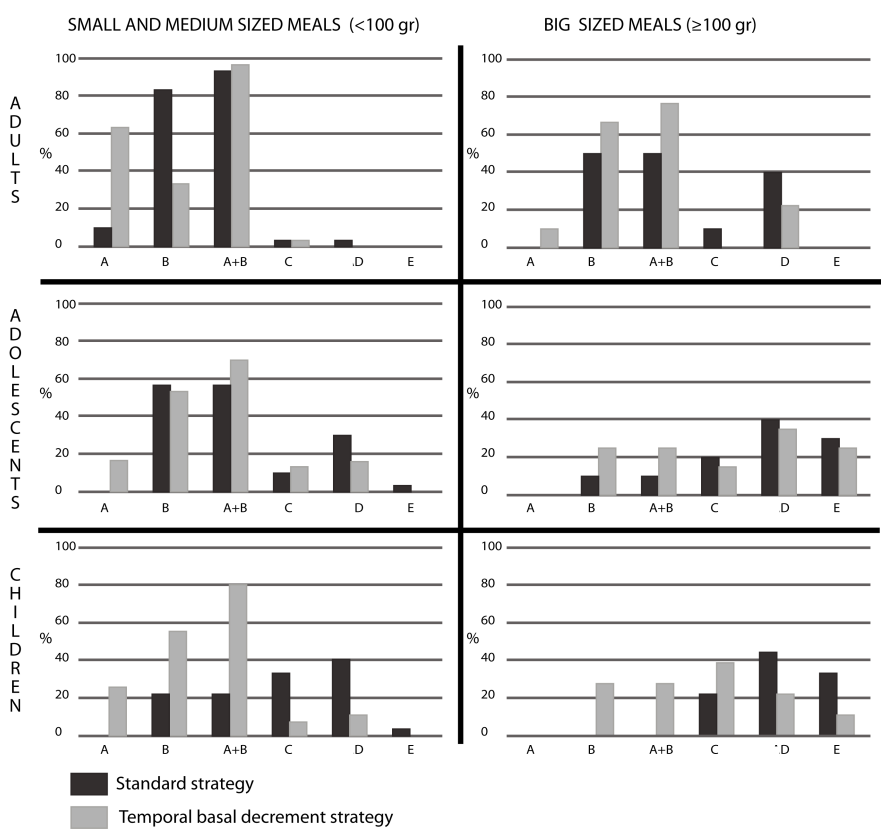

Fig. 11. Histograms showing the number of points falling in each of the CVGA zones. The dark bars represent the results with the standard bolus administration mode whereas the light bars correspond to the temporal basal decrement strategy. A seven-hour time horizon has been used.

\section{CONClusion}

In this contribution, an algorithm for calculating the most appropriate combination of basal and bolus insulin for a good postmeal glucose control is thoroughly presented. Set inversion methods based on interval analysis are applied to determine, for a given meal, which bolus administration mode will yield a glucose response fulfilling the IDF guidelines of postprandial control.

The theoretical basis of the algorithm are detailed, and an in silico validation with the adults, adolescents and children in the UVa simulator is presented, demonstrating 
robustness with respect to the patient model. Generally, for intermediate-size meals, either a dual-wave bolus or a temporal basal decrement is suitable. However, for higher meals, only temporal basal decrement can yield a good postprandial control. Clinical trials to evaluate the efficacy of the algorithm in vivo are currently ongoing.

It is proved that for high meals, the current standard therapy consisting of a bolus insulin on top of basal for controlling glucose after a meal, is not enough for assuring a good glucose performance. Alternative, coordinating basal and bolus insulin can improve significantly this performance.

\section{ACKNOWLEDGMENT}

The authors acknowledge the support of the Spanish government under the grants DPI-2007-66728, the European Union through FEDER funds, Generalitat de Catalunya under grant SGR-00296 and Universidad Politécnica de Valencia through the PAID-05-08 programme. The authors acknowledge the support of Howard Zisser from Sansum Diabetes Research Institute and Francis J. Doyle III and Eyal Dassau from University of California Santa Barbara in this research line.

\section{REFERENCES}

[1] Diabetes Control and Complications Trial Research Group, "The effect of intensive treatment of diabetes on the development and progression of long-term complications in insulindependent diabetes mellitus." The New England Journal of Medicine, vol. 329, pp. 977-986, 1993.

[2] H. P. Chase, S. Z. Saib, T. MacKenzie, M. M. Hansen, and S. K. Garg, "Post-prandial glucose excursions following four methods of bolus insulin administration in subjects with type 1 diabetes," Diabetic Medicine, vol. 19, no. 4, pp. 317-321, 2002.

[3] J. Bondia, E. Dassau, H. Zisser, R. Calm, J. Vehí, L. Jovanovic, and F. J. Doyle III, "Coordinated basal-bolus infusion for tighter postprandial glucose control in insulin pump therapy," Journal of Diabetes Science and Technology, vol. 3, no. 1, pp. 89-97, 2009.

[4] International Diabetes Federation, "Guideline for management of postmeal glucose." [Online]. Available: http://www.idf.org/webdata/docs/Guideline_PMG_final.pdf

[5] B. P. Kovatchev, M. Breton, C. Dalla Man, and C. Cobelli, "In silico preclinical trials: A proof of concept in closed-loop control of type 1 diabetes," Journal of Diabetes Science and Technology, vol. 3 , no. 1 , pp. $44-55,2009$.

[6] R. E. Moore, Interval analysis. Englewood Cliffs, N.J.: Prentice-Hall Inc., 1966.

[7] R. E. Moore, Methods and Applications of Interval Analysis. SIAM Studies in Applied and Numerical Mathematics, 1979.

[8] G. Alefeld and J. Herzberger, Interval analysis. Academic Press, 1983.

[9] L. Jaulin, M. Kieffer, O. Didrit, and E. Walter, Applied Interval Analysis. Springer, 2001.

[10] M. García-Jaramillo, R. Calm, J. Bondia, C. Tarín, and J. Vehí, "Computing the risk of postprandial hypo- and hyperglycemia in. type 1 diabetes mellitus considering intrapatient variability and other sources of uncertainty," Journal of Diabetes Science and Technology, vol. 3, no. 4, pp. 895-902, 2009.

[11] E. Walter and L. Pronzato, Identification of parametric models from experimental data. Communications and Control Engineering Series. Berlin: Springer. xviii, 413 p., 1997.

[12] L. Ljung, System Identification: Theory for the User (2nd Edition). Prentice Hall PTR, December 1999.

[13] R. Hovorka, V. Canonico, L. J. Chassin, U. Haueter, M. MassiBenedetti, M. O. Federici, T. R. Pieber, H. C. Schaller, L. Schaupp, T. Vering, and M. E. Wilinska, "Nonlinear model predictive control of glucose concentration in subjects with type 1 diabetes," Physiol Meas, vol. 25, no. 4, pp. 905-920, 2004.
[14] J. Walsh, "Changes in diabetes care: a history of insulin and pumps - past, present, and future." 2004. [Online]. Available: http://www.childrenwithdiabetes.com/presentations/DMCarePast-Future-0904_files/v3_document.htm

[15] J. Walsh and R. Roberts, Pumping insulin: everything you need for success on a smart insulin pump., 4th ed. San Diego: Torrey Pines Press., 2006.

[16] L. Magni, D. M. Raimondo, C. D. Man, M. Breton, S. Patek, G. D. Nicolao, C. Cobelli, and B. P. Kovatchev, "Evaluating the efficacy of Closed-Loop glucose regulation via ControlVariability grid analysis," Journal of Diabetes Science and Technology, vol. 2, no. 4, pp. 630-635, 2008.

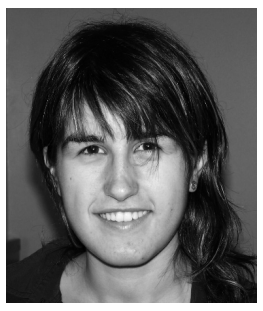

A. Revert received the M.S.Eng. degree from the Universidad Politécnica de Valencia, Spain in 2008. She is currently a PhD student at this same university. Her main research interests are in modeling and control in type 1 diabetes and systems biology.

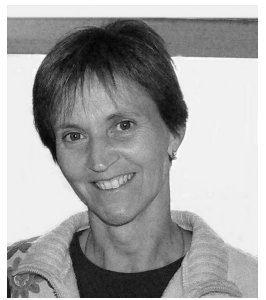

R. Calm received the $\mathrm{PhD}$ degree in Mathematics from the Technical University of Catalonia, UPC (Spain) in 2005. Since 1992 she has been Lecturer Professor at the Department of Computer Science and Applied Mathematics of the University of Girona. Her main research interests are in the field of modal interval analysis and their applications to modeling and control of biomedical systems.

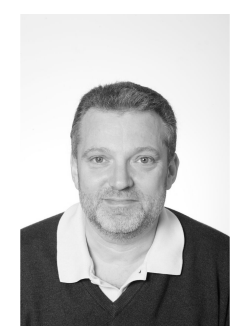

J. Vehí (M'97) received the Doctoral degree in Electrical Engineering in 1998 from the University of Girona, Spain. He was Teaching Assistant, from 1987 to 1992 at the Technical University of Catalonia, Spain. From 1992 to 1999 he was Assistant professor at the University of Girona. In 1999 he became Associate Professor of Electrical Engineering at the University of Girona. Since 2006 he is the director of the Research Institute on Computer Engineering and Automation at the University of Girona. His present research interests include Interval methods for control, modeling and control of biomedical systems, robust and nonlinear control, fault detection and diagnostics for complex dynamic systems and intelligent methods for structural assessment.

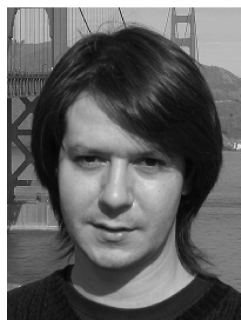

J. Bondia M.S.'94 and PhD'02 in Computer Science from Universidad Politécnica de Valencia, Spain. In 1995 he starts teaching at the Department of Systems Engineering and Control of this same university, holding the position of Associate Professor since 2007. His main research interests are modelling and control in type 1 diabetes, biomedical applications, systems biology, uncertain dynamical systems, interval analysis and monotone systems. 